\title{
Intravesical botulinum toxin for lower urinary tract dysfunction
}

\section{Marcus John Drake}

Address: Bristol Urological Institute, Southmead Hospital, Bristol, BS10 5NB, UK

Email: marcus.drake@bui.ac.uk

Fl000 Medicine Reports 2010, 2:6 (doi:10.34I0/M2-6)

The electronic version of this article is the complete one and can be found at: http://f1000.com/reports/medicine/content/2/6

\begin{abstract}
Botulinum neurotoxin has achieved substantial clinical benefits in neurogenic detrusor overactivity. More contentious has been its extension to management of refractory idiopathic detrusor overactivity, in which risk of impaired voiding function and consequent need for intermittent catheterisation may counteract the quality-of-life benefits of improved storage function. Several issues remain uncertain for this unlicensed treatment, and clear insights into long-term consequences are needed before it can be regarded as an established mainstream treatment.
\end{abstract}

\section{Introduction and context}

In the context of lower urinary tract (LUT) dysfunction, botulinum neurotoxin-A (BoNT-A) was first used to treat neurogenic bladder caused by spinal cord injury. The substantial improvement in symptom severity achieved represented a clear advance in clinical management options, and benefits in other neurological conditions such as multiple sclerosis were subsequently documented. The benefits relate to improved LUT storage function as exemplified by reduced amplitude of detrusor overactivity (DO) and incontinence severity. In neurogenic LUT dysfunction, intravesical BoNT-A treatment carries a significant likelihood of further impairing voiding efficiency, ranging from voiding symptoms through raised post-void residual to inability to pass urine. Accordingly, the majority of patients with neurogenic LUT dysfunction require intermittent catheterisation (IC). IC is commonly employed as a management option in this patient group as a consequence of voiding dysfunction resulting from the neurological disease and is generally well tolerated (perhaps because of neurogenic sensory impairment affecting the urethra). Thus, additional impairment of voiding efficiency resulting from intravesical BoNT-A treatment has not generally been regarded as a contraindication to its use. Clinical uptake of the method has been widespread, with two key issues outstanding: (a) the need for regulatory approval, as the technique is currently unlicensed, and (b) the need for long-term studies demonstrating that improved LUT storage parameters translate into preservation of upper urinary tract function.

The success of intravesical BoNT-A treatment in the neurogenic context has led to extrapolation to idiopathic DO. This is more contentious for several reasons, for example;

1. Indications for intervention. There is no compelling medical reason to intervene since preservation of upper urinary tract function is rarely a consideration. Thus, treatment is primarily aimed at improving quality of life, yet exchanging storage failure for voiding failure is not necessarily regarded as an improvement by the individual involved. Most studies have undertaken urodynamic tests to evaluate filling and voiding function before injection, and most published research has been based on patients with idiopathic DO. Where patients have idiopathic overactive bladder (OAB) symptoms (as defined by symptomatic complaint of urinary urgency) but do not have DO when assessed via urodynamics, less information is available. Although benefits have been reported [1], the use of BoNT-A in this group requires particular care. Key markers for predicting response to treatment have not been identified thus far [2], except that a high maximum detrusor pressure may predict poor treatment outcome [3]. 
2. Risk of inducing voiding dysfunction. IC is not in widespread use in this group of patients, and the potential emergence of voiding dysfunction as a consequence of treatment effectively leaves the patient faced with a choice between having to tolerate refractory storage failure or replacing it with de novo voiding failure. Risk of needing IC postoperatively varies widely (reviewed in [4]). It is thus prudent to inform patients fully about the potential consequences of proceeding and to train them in IC prior to injection since some patients find themselves unable to carry out the procedure. The ability to predict which patients are most at risk of developing voiding dysfunction would be advantageous. Urodynamically, projected isovolumetric pressure in women and bladder contractility index in men may be relevant [5], but further information is needed.

3. Risk of systemic adverse effects. The number of side effects that have been reported is low, but the need for safety measures is emphasized. In April 2009, the American regulatory authorities issued guidance on the risks of serious adverse events associated with systemic spread of the toxin [6]. They emphasized that physicians need to warn patients about the potential for effects following administration of botulinum toxins such as unexpected loss of strength or muscle weakness, hoarseness or trouble talking, trouble saying words clearly, loss of bladder control, trouble breathing, trouble swallowing, double vision, blurred vision, and drooping eyelids. These have been reported as early as several hours and as late as several weeks after treatment, and patients need to be advised to seek immediate medical attention if they develop any of these symptoms. Health care professionals and consumers can report serious adverse events through the US Food and Drug Adminstration's MedWatch Adverse Event Reporting Program.

4. Regimes of administration. Approaches to administering treatment are not currently standardised. Dose, depth of injection (into the detrusor or suburothelial), bladder region (whether or not the trigone is injected and even the prostate gland in men [7]), anaesthesia, and BoNT-A brand (BoTox or Dysport) are each sources of variation which might influence the interpretation of outcomes. Results of large-scale licensing trials which may eventually inform a more standardised approach (depending on outcome) are awaited. More information is needed before this realistically can be anticipated [8].

5. Duration of initial response and response to repeat treatment. Efficacy is not as well sustained in idiopathic DO as in neurogenic DO; duration of improved symptom severity varies between studies, but 6 months is a suitable ballpark figure to quote to patients. A substantial proportion of patients see a return of symptom control when injections are repeated, but not all do so. Accordingly, it is not appropriate to expect lifelong symptom control in this group of patients until long-term follow-up studies have been completed. This information is a long way off and needs to identify key parameters, such as subgroups standing the best chance of sustained response, whether risk of voiding dysfunction increases, whether functional behaviour of the urinary tract deteriorates (e.g., filling compliance of the bladder) and whether quality-of-life improvement is sustained. Even in neurogenic DO, which is regarded as having a better initial response and a good prospect with repeat injection, the longer-term response is down to $74 \%$ at the fourth administration and, worryingly, with some suggestion that detrusor contractility does not recover fully [9].

6. Health economics. Treatment purchasers tend to regard funding of treatments aimed at quality-of-life improvement as a lower priority. Studies demonstrating costeffectiveness and cost utility will be needed to establish intravesical BoNT-A treatment in health service provision. The treatment is expensive in relation to drug purchase, treatment delivery, repeated treatment and proportionate need for provision of catheters for IC. While savings can be anticipated from the reduced use of incontinence containment products and antimuscarinic pharmaceuticals, real benefits for quality of life and social/employment function will be essential before health economics favour supporting provision of the treatment method.

The pressing need for larger placebo-controlled and comparative trials to evaluate the efficacy of single and repeat injections, the duration of effect, the optimal dosage and injection technique, the timing for repeat injection, and the short- and long-term safety of the treatment in LUT and pelvic floor disorders is clearly emphasized by the medical profession [10].

\section{Recent advances}

1. Some work is suggesting that lower doses of BoNT-A can be effective for symptom control (for example, [11]) in idiopathic DO. This is still not established, nor is longevity of response or whether repeated injections achieve equivalent symptom improvement.

2. The mechanisms of action involved may be more diverse than impairment of acetylcholine release from motor nerves. Sensory parameters (e.g., urgency severity) appear to be affected rapidly [12], and altered neuropeptide release has been reported in animal models [13]. 
This may offer indirect support for an afferent basis to $\mathrm{OAB}$ pathophysiology and response to BoNT-A.

3. In neurogenic DO, symptom benefits and improvement in urodynamic parameters have been reported in a more diverse group of patients, including those with Parkinson's disease [14] and multiple sclerosis [15].

4. Use in situations outside $\mathrm{DO}$ and $\mathrm{OAB}$, including bladder neck dyssynergia [16], urogenital pain syndromes (e.g., [17]) and acute urinary retention in men [18], has been attempted. Nonetheless, these small-scale studies should be regarded as providing interesting priming data for future research rather than serving as a basis for clinical management.

5. Altering the molecular structure and related proteins of the administered agent may represent a strategy to modulate the balance of efficacy and unwanted effects, potentially strengthening the case for long-term clinical treatment. Animal studies of at least one such formulation have been undertaken [19]. Additionally, combining BoNT-A with intravesical instillation of dimethyl sulphoxide to administer the agent without the need for injection has been reported [20].

\section{Implications for clinical practice}

Intravesical BoNT-A treatment is becoming widely accepted in the medical community for the treatment of symptoms caused by neurogenic DO. It remains unlicensed as yet, but the clinical trials required for regulatory approval are in progress. Certainly, many patients experience better storage function when intravesical BoNT-A treatment is combined with IC, and this has been recognised (e.g., the UK statement on bladder management in multiple sclerosis [21]). Key issues in this context include the potential contribution of the treatment in maintaining a 'safe bladder' [i.e., one that will not endanger upper urinary tract function due to reduced filling compliance, DO in the presence of vesicoureteric reflux, or detrusor sphincter dyssynergia (DSD)]. It is crucial that BoNT-A treatment sustain the improved life expectancy in neurogenic bladder achieved through better protection of renal function over the last decades. Clinicians are also using BoNT-A for additional benefits, such as sphincter injections in DSD and reduction of bladder spasms in patients with indwelling urinary catheters.

Idiopathic DO is less clear-cut, and clinicians need to consider carefully whether they are exposing patients to potential problems. In the immediate aftermath, the majority of patients manage to void adequately, but the difficulty predicting who will need IC means the patient must fully understand the risk and precisely what IC entails. Short-term response rates in idiopathic DO are lower than in neurogenic DO and quality-of-life improvement is also less clear-cut. In the medium term, there is some suspicion that detrusor contractility may be impaired, potentially leading to increased numbers of patients needing IC. The longer term entails even greater uncertainty; is it really realistic to anticipate lifelong treatment at 6-month intervals? What is the attrition rate of treatment response? What will be the severity of the symptoms: improved as the bladder is trained to cope with greater volumes or increased as neural plasticity alters the pathophysiology of the condition?

Besides the interest for BoNT-A, other treatments for refractory DO such as sacral nerve stimulation are less used and seem to be less known by the urological community and general practitioners. However, this treatment has received a grade A recommendation for refractory DO from the fourth International Consultation on Incontinence. Patients should be informed not only about the different options for their refractory $\mathrm{OAB}$ symptoms but also about the level of evidence for these treatments in order to enable them to make a properly informed decision.

\section{Conclusions}

Intravesical BoNT-A treatment can achieve many benefits, but refinement of the protocols and clarification of long-term effects are essential in both neurogenic and idiopathic DO. Use of the treatment is acceptable only where patients are fully counselled as to uncertainty and risk and where clinicians maintain close surveillance of their treated patients and keep aware of scientific developments. Further research is needed clinically, but at the basic science level, research into the mechanisms underlying clinical response may yield information on potential targets for the development of new treatment approaches.

\section{Abbreviations}

BoNT-A, botulinum neurotoxin-A; DO, detrusor overactivity; DSD, detrusor sphincter dyssynergia; IC, intermittent catheterisation; LUT, lower urinary tract; $\mathrm{OAB}$, overactive bladder.

\section{Competing interests}

The author has provided support for basic science research, served on advisory boards and fulfilled speaker engagements for Astellas Pharma (Tokyo, Japan) and provided support for clinical research, served on advisory boards and fulfilled speaker engagements for Pfizer Inc. (New York, NY, USA). 


\section{References}

I. Flynn MK, Amundsen CL, Perevich M, Liu F, Webster GD: Outcome of a randomized, double-blind, placebo controlled trial of botulinum A toxin for refractory overactive bladder. J Urol 2009, | 81:2608-|5.

2. Cohen BL, Caruso DJ, Kanagarajah P, Gousse AE: Predictors of response to intradetrusor botulinum toxin-a injections in patients with idiopathic overactive bladder. Adv Urol 2009: 328-64.

3. Sahai A, Khan MS, Le Gall N, Dasgupta P: Urodynamic assessment of poor responders after botulinum toxin-A treatment for overactive bladder. Urology 2008, 71:455-9.

4. Shaban AM, Drake MJ: Botulinum toxin treatment for overactive bladder: risk of urinary retention. Curr Urol Rep 2008, 9:445-5 I.

5. Sahai A, Sangster P, Kalsi V, Khan MS, Fowler CJ, Dasgupta P: Assessment of urodynamic and detrusor contractility variables in patients with overactive bladder syndrome treated with botulinum toxin-A: is incomplete bladder emptying predictable? BJU Int 2009, I03:630-4.

FI000 Factor 3.0 Recommended

Evaluated by Marcus Drake 02 Jan 2009

6. US Food and Drug Administration: FDA requires boxed warning for all botulinum toxin products; 30 April 2009. [http://www.fda. gov/NewsEvents/Newsroom/PressAnnouncements/ucm I 49574.htm]

7. Kuo HC: Prostate botulinum A toxin injection-an alternative treatment for benign prostatic obstruction in poor surgical candidates. Urology 2005, 65:670-4.

8. Karsenty G, Denys P, Amarenco G, De Seze M, Game X, Haab F, Kerdraon J, Perrouin-Verbe B, Ruffion A, Saussine C, Soler JM, Schurch B, Chartier-Kastler E: Botulinum toxin A (Botox) intradetrusor injections in adults with neurogenic detrusor overactivity/neurogenic overactive bladder: a systematic literature review. Eur Urol 2008, 53:275-87.

9. Pannek J, Gocking K, Bersch U: Long-term effects of repeated intradetrusor botulinum neurotoxin $\mathrm{A}$ injections on detrusor function in patients with neurogenic bladder dysfunction. BJU Int 2009, I 04: 1 246-50.

10. Apostolidis A, Dasgupta P, Denys P, Elneil S, Fowler CJ, Giannantoni A, Karsenty G, Schulte-Baukloh H, Schurch B, Wyndaele JJ: Recommendations on the use of botulinum toxin in the treatment of lower urinary tract disorders and pelvic floor dysfunctions: a European consensus report. Eur Urol 2008, [Epub ahead of print].

FI000 Factor 3.0 Recommended

Evaluated by Dirk De Ridder 23 Feb 2009
II. Cohen BL, Barboglio P, Rodriguez D, Gousse AE: Preliminary results of a dose-finding study for botulinum toxin-A in patients with idiopathic overactive bladder: 100 versus 150 units. Neurourol Urodyn 2009, 28:205-8.

12. Kalsi V, Apostolidis A, Gonzales G, Elneil S, Dasgupta P, Fowler Cl: Early effect on the overactive bladder symptoms following botulinum neurotoxin type $A$ injections for detrusor overactivity. Eur Urol 2008, 54:18I-7.

13. Lucioni A, Bales GT, Lotan TL, McGehee DS, Cook SP, Rapp DE: Botulinum toxin type $A$ inhibits sensory neuropeptide release in rat bladder models of acute injury and chronic inflammation. BJU Int 2008, I0 I:366-70.

FI000 Factor 3.0 Recommended Evaluated by Rodolfo Testa 17 Jan 2008

14. Giannantoni A, Rossi A, Mearini E, Del Zingaro M, Porena M, Berardelli A: Botulinum toxin $\mathbf{A}$ for overactive bladder and detrusor muscle overactivity in patients with Parkinson's disease and multiple system atrophy. J Urol 2009, I 82:|453-7.

15. Jabbari B: Botulinum toxin A: a new treatment option for multiple-sclerosis-related bladder overactivity? Nat Clin Pract Neurol 2008, 4:188-9.

16. Lim SK, Quek PL: Intraprostatic and bladder-neck injection of botulinum $A$ toxin in treatment of males with bladder-neck dyssynergia: a pilot study. Eur Urol 2008, 53:620-5.

17. Giannantoni A, Porena M, Costantini E, Zucchi A, Mearini L, Mearini E: Botulinum A toxin intravesical injection in patients with painful bladder syndrome: I-year followup. J Urol 2008, | 79: $103 \mid-4$

18. Silva J, Silva C, Saraiva L, Silva A, Pinto R, Dinis P, Cruz F: Intraprostatic botulinum toxin type a injection in patients unfit for surgery presenting with refractory urinary retention and benign prostatic enlargement. Effect on prostate volume and micturation resumption. Eur Urol 2008, 53:I53-9.

19. Nishiyama Y, Yokoyama T, Tomizawa K, Okamura K, Yamamoto Y, Matsui $H$, Oguma K, Nagai A, Kumon H: Effects of purified newly developed botulinum neurotoxin type $A$ in rat prostate. Urology 2009, 74:436-9.

20. Petrou SP, Parker AS, Crook JE, Rogers A, Metz-Kudashick D, Thiel DD: Botulinum a toxin/dimethyl sulfoxide bladder instillations for women with refractory idiopathic detrusor overactivity: a phase I/2 study. Mayo Clin Proc 2009, 84:702-6.

21. Fowler CJ, Panicker JN, Drake M, Harris C, Harrison SC, Kirby M, Lucas M, Macleod N, Mangnall J, North A, Porter B, Reid S, Russell N, Watkiss K, Wells M: A UK consensus on the management of the bladder in multiple sclerosis. I Neurol Neurosurg Psychiatry 2009, 80:470-7. 